\title{
28 Research Square \\ Predicting successful lumbar plexus block using blood flow index measured by laser speckle contrast imaging
}

\section{Xi Wu}

Tongji Hospital of Tongji Medical College of Huazhong University of Science and Technology

\section{Pengcheng Li}

Huazhong University of Science and Technology

Xiaohu Liu

Huazhong University of Science and Technology

\section{Liangwei Meng}

Huazhong University of Science and Technology

\section{Dan Wang}

Tongji Hospital

\section{Yujie Huang}

Tongji Hospital

Wei Mei ( $\nabla$ wmei@hust.edu.cn )

Tongji Hospital https://orcid.org/0000-0001-6556-6628

\section{Research article}

Keywords: lasers, nerve block, regional blood flow, ultrasonography

Posted Date: September 16th, 2019

DOI: https://doi.org/10.21203/rs.2.14429/v1

License: (9) This work is licensed under a Creative Commons Attribution 4.0 International License. Read Full License 


\section{Abstract}

Background: Laser speckle contrast imaging (LSCI) is a powerful optical imaging technique for real-time and dynamic measurement of regional blood flow. The objective of this prospective observational study was to investigate blood flow after changes blockade of lumbar plexus or its three major branches respectively, with $\mathrm{LSCl}$ technique.

Methods: This study included 47 adult patients scheduled for elective lower limb surgery. For the selective blockade of lumbar plexus, femoral nerve, obturator nerve, and lateral femoral cutaneous nerve, blood flow images and pinprick sensory scores of the blocked lower limb were recorded 5 min pre block and every 5 min for 30 min post block. Blood flow index (BFI) values of toes were calculated by LSCI software.

Results: In this study, we have 21 cases of successful lumbar plexus blocks, 2 cases of failed lumbar plexus block, 8 cases of successful femoral nerve blocks, 8 cases of successful obturator nerve blocks, and 8 cases of successful lateral femoral cutaneous nerve blocks. The BFI values of all five toes were significantly increased as early as $5 \mathrm{~min}$ after successful lumbar plexus block, whereas no significant difference was found in BFI values after failed lumbar plexus block. BFI changes after successful selective blockade of femoral nerve, obturator nerve, or lateral femoral cutaneous nerve were negligible. BFI value of the big toe at 5 min after the successful lumbar plexus block was increased by 2.57 fold compared with the baseline value, which represented the highest increase among five tested toes. BFI value of the big toe at 10 min after lumbar plexus block showed great power to predict block outcome with a sensitivity of $100 \%$ and a specificity of $100 \%$. The optimal cut-off value given by ROC analysis was 22.11 PU.

Conclusions: Increased blood flow index measured by laser speckle contrast imaging is a reliable indicator of successful lumbar plexus block, but cannot indicate successful selective blocks of three major branches of the lumbar plexus. BFI value of the big toe at 10 min after lumbar plexus block could well predict block outcome with the cut-off value of $22.11 \mathrm{PU}$.

Trial registration: NCT03169517 (Date of registration: May 25, 2017).

\section{Background}

Lumbar plexus block is a useful technique in the management of pain after lower extremity surgery. ${ }^{1-3}$ Traditional assessment methods of block success, such as pinprick and cold sensory tests, rely on patient compliance and individual subjective judgment. There is a need for objective methods to evaluate the quality of nerve block. Successful peripheral nerve block could induce local vasodilation and increase blood flow via sympathetic blockade. ${ }^{45}$ The success of lumbar sympathetic block can be assessed by various measurements, such as laser Doppler perfusion imager, thermometry, infrared thermography, and pulse oximetry. ${ }^{6-10}$ However, these tests are subject to poor temporal resolution and low spatial resolution because of long time intervals of data acquisition and limited observation field. Laser speckle 
contrast imaging (LSCI) is an optical imaging technique that allows real-time and full-field assessment of blood flow with high spatial and temporal resolution. ${ }^{11-13} \mathrm{LSCl}$ has been widely applied in monitoring cerebral blood flow, skin perfusion, and retinal blood flow. ${ }^{14-19}$ Our previous study demonstrated that LSCI might be an early, objective, quantitative, and reliable indicator of successful sciatic block. ${ }^{20}$ The primary aim of this study was to investigate blood flow changes after lumbar plexus block using LSCI technique.

The three major branches of the lumbar plexus are the femoral nerve, the obturator nerve, and the lateral femoral cutaneous nerve. It was reported that changes of skin temperature could predict the success of infraclavicular brachial plexus block. ${ }^{21}$ Selective blockade of the ulnar and median nerve resulted in substantial increases in skin temperature of the hand, whereas selective blocking of the musculocutaneous or radial nerve did not increase skin temperature in any area. ${ }^{22}$ This may imply that blockade of the proximal trunk and distal branches of the nerve may have different impact on sympathetic fibers. The blood flow changes after selective blockade of the femoral nerve, the obturator nerve, or the lateral femoral cutaneous nerve have never been studied. The secondary aim of this study was to explore the changes of blood flow after selective blockade of three major branches of the lumbar plexus.

\section{Methods}

This prospective observational study was approved by Ethics Committee of Tongji Hospital (TJIRB20170401) and written informed consent was obtained from all subjects participating in the trial. The trial was registered prior to patient enrollment at clinicaltrials.gov (NCT03169517, Principal investigator: Wei Mei, Date of registration: May 25, 2017). In this study, 47 patients scheduled for elective lower limb surgery between May 2017 and September 2017 at Tongji Hospital in Wuhan, China were enrolled. Inclusion criteria were American Society of Anesthesiologists (ASA) physical status I-II and age 18 to 75 years. Exclusion criteria were peripheral vascular disease, toe injury or deficiency, colored or infected toenails, vasodilator medications prior to surgery, preoperative analgesic intake, body mass index $>35$, or contraindication to peripheral nerve block (including patient refusal, allergy to local anesthetics, peripheral neuropathy, or coagulopathy).

Upon arrival at the anesthesia induction room, intravenous access and routine monitoring with electrocardiography, noninvasive blood pressure, and pulse oximetry were performed. All patients rest quietly in supine position and were allowed $20 \mathrm{~min}$ to adapt to the surroundings before measurement. Room temperature was kept at $24^{\circ} \mathrm{C}$. Blood flow images of the toes in the operated limb were obtained by $\mathrm{LSCl}$ and pinprick sensory tests were performed 5 min before nerve block $(t=0)$ as the baseline value. Ultrasound guidance was performed using a low-frequency ( 2 to $5 \mathrm{MHz}$ ) curved array transducer (SonoSite M-Turbo; SonoSite Inc, Bothell, Washington) for lumbar plexus block and a high-frequency (6 to $13 \mathrm{MHz}$ ) linear array probe (SonoSite M-Turbo; SonoSite Inc, Bothell, Washington) for blockade of the femoral nerve, the obturator nerve, and the lateral femoral cutaneous nerve. An 21-gauge, 10-cm Tuohy 
needle (UniPlex Nanoline; Pajunk Inc, Geisingen, Germany) was used for nerve block in our study. All the blocks were performed by a dedicated investigator (W. M.).

For lumbar plexus block, patients were turned to the lateral decubitus position with the side to be blocked upward. Ultrasound-guided lumbar plexus block was performed by a paramedian transverse scan (PMTS) of the lumbar paravertebral region with the ultrasound beam being insonated through the intertransverse space (ITS) (PMTS-ITS). ${ }^{23}$ The lumbar plexus nerve was identified as a hyperechoic structure in the posterior aspect of the psoas muscle. After infiltration with $1 \%$ lidocaine, the needle connected to a nerve stimulator (Stimuplex, HNS 12, Braun Medical, Melsungen, Germany) was placed lateral to the probe and slowly advanced toward the lumbar plexus nerve using an in-plane technique. Correct needle position was confirmed when ipsilateral quadriceps muscle contraction was elicited at a current of $0.5-0.8 \mathrm{~mA}$. Subsequently, $30 \mathrm{ml}$ of $0.4 \%$ ropivacaine was administered with repeated negative aspiration. After the completion of lumbar plexus block, patients were returned to the supine position.

For femoral nerve block (FNB), patients were placed in supine position. The ultrasound probe was placed inferior to the inguinal ligament and the femoral nerve was appeared as an oval hyperechoic structure which was lateral to femoral artery. ${ }^{24}$ Insert the needle from the lateral edge of the probe with an in-plane approach. The trace and the tip of the needle could be well visualized as it approached the target nerve. Correct needle position was confirmed when $2 \mathrm{ml}$ of saline spread as an expanding hypoechoic area surrounding the nerve. Subsequently, $15 \mathrm{ml}$ of $0.4 \%$ ropivacaine was injected with repeated negative aspiration.

For obturator nerve block (ONB), patients lay supine with the hip externally rotated. The transducer was positioned at the proximal thigh inferior to the inguinal ligament. The anterior division was seen as a hyperechoic and oval structure between the adductor longus and brevis muscles. The posterior division was between the adductor brevis and magnus muscles. ${ }^{24}$ With in-plane technique, the needle was first directed into the fascial plane between the adductor brevis and magnus muscles and $5 \mathrm{ml}$ of $0.4 \%$ ropivacaine was injected at this location. Then the needle was withdrawn to the superficial plane between the adductor longus and brevis muscles and $5 \mathrm{ml}$ of $0.4 \%$ ropivacaine was administered.

For lateral femoral cutaneous nerve block (LFCNB), patients were placed supine. The linear probe was placed on the inguinal crease and moved towards the anterior superior iliac spine. The lateral femoral cutaneous nerve was appeared as hyperechoic structure between the fascia lata and iliaca. ${ }^{24}$ Insert the needle in a lateral-to-medial direction with in-plane approach. Subsequently, $5 \mathrm{ml}$ of $0.4 \%$ ropivacaine was injected with repeated negative aspiration.

Blood flow images by LSCI and pinprick sensory scores were recorded every $5 \min (t=5, t=10, t=15, t=$ $20, t=25, t=30)$ for 30 min starting from the completion of local anesthetics injection. At each time point, LSCl monitoring was performed prior to pinprick sensory tests. Pinprick sensory tests were performed with a 22 gauge blunt needle on the skin innervated by the femoral nerve (anterior aspect of the midthigh), obturator nerve (medial aspect of the midthigh), and lateral femoral cutaneous nerve 
(lateral aspect of the midthigh). ${ }^{2526}$ The pinprick sensory measurement was performed bilaterally with the same pinprick stimulus. The rating scale for pinprick sensory blockade was 1 (sensation) and 0 (no sensation). Blood flow images and pinprick sensory test scores were obtained individually by two investigators for each patient (LSCl: X. W.; pinprick sensory tests: D. W.). The two investigators were blinded to each other's assessments. Haemodynamic data including non-invasive blood pressure, heart rate, and oxygen saturation were also recorded at each time point.

Blood flow index (BFI) was measured by a laser speckle blood flow imaging system ${ }^{15}$ (SIM BFI-WF, SIM Opto-Technology Co., Ltd., Wuhan, China). The equipment mainly consists of a wheeled stage, a laser speckle imaging head, and a connection arm that enables the imaging head moving flexibly in horizontal and vertical directions. The system uses a laser beam with the wavelength of $785 \mathrm{~nm}$ to illuminate the target area and employs a 12-bit charge-coupled device (CCD) camera to acquire the speckle pattern. The CCD exposure time was set to $20 \mathrm{~ms}$. The light was perpendicular to the patient's foot at a measurement distance of $20-25 \mathrm{~cm}$. Thirty consecutive images were acquired at an interval of $1 \mathrm{~s}$ and Region of interests (ROIs) could be selected in the blood flow image. Mean BFI values of the ROIs were calculated by LSCI software (SIM BFI Software, SIM Opto-Technology Co., Ltd., Wuhan, China), which expresses values in perfusion unit (PU). In our study, five separate ROls (circular areas of $1 \mathrm{~cm}$ in diameter on the nail bed of each toe) were established by the circular-ROI selecting tool in the software (Fig. 1)..

Lumbar plexus block was defined as successful if complete loss of sensation was observed in all 3 nerve distribution areas at $30 \mathrm{~min}$ after the block. In contrast, lumbar plexus block was identified as failed when pinprick test in any of the 3 nerve innervation areas was recorded as "sensation" at 30 min post block. Successful FNB, ONB, and LFCNB were respectively defined as "no sensation" in the anterior, medial, and lateral aspect of the midthigh at 30 min after the block. On the contrary, failed FNB, ONB, and LFCNB were identified as sensation still existing in the nerve innervating area at 30 min post block.

\section{Statistical Analysis}

Sample size was calculated by MedCalc Software version 15.2 (MedCalc Software, Ostend, Belgium). This study was originally designed to detect differences in BFI values after nerve block compared with the pre-block baseline value. Based on our previous study, the mean difference of BFI value was 42.77 PU and the standard deviation was $21.18 \mathrm{PU}$. Setting the a-level to be 0.05 and the power to be $90 \%$, the sample size required for the comparison of two paired samples was calculated as 5 . To make the results more convincing, we enrolled at least 8 patients for each type of nerve block. We performed statistical analyses by SPSS 22.0 (IBM Corp., New York, USA) and made all line graphics with GraphPad Prism version 6.01 for Windows (Graph Pad Software, San Diego, CA). Continuous data are expressed as mean \pm SD or median (IQR). Categorical data are reported as number (percentage). The independent sample $t$ test was used for between-group comparisons of normally distributed data. Categorical data were compared using the Fisher exact test. Repeated-measures analysis of variance (ANOVA) was applied to compare measurements over time. If significant, a two-sided paired $t$ test was performed to examine 
differences at individual time points within each group. A $P$ value of less than 0.05 was considered significant. A receiver operator characteristic (ROC) curve analysis was used for discriminating the power of the increased blood flow index to detect a successful block.

\section{Results}

During the study period, 47 patients completed the study. Lumbar plexus block was successful in 21 patients and failed in 2 patients. There were 8 cases of successful FNB, 8 cases of successful ONB, and 8 cases of successful LFCNB in our study. Surgeries performed included knee arthroscopy, total knee arthroplasty, internal fixation of femoral fracture, femoral plate removal, femur lesion excision, total hip replacement, femoral drilling decompression, and adductor myotomy. Patient characteristics are shown in Table 1. There were no significant demographic differences in patients with successful and failed lumbar plexus block.

As shown in Fig. 2, BFI value at each toe increased sharply within 5 min after the successful lumbar plexus block. BFI value at the fourth toe was peaked at 5 min after the successful lumbar plexus block while BFI values at the other toes were peaked at 30 min after the block. For successful lumbar plexus block, BFI values at each time point after the block were significantly higher than the baseline value at all tested toes $(P \leq 0.001)$. BFI value of the big toe at $5 \mathrm{~min}$ after the successful lumbar plexus block was increased by 2.57 fold compared with the baseline value, which represented the highest increase among five tested toes. At 5 min after the successful lumbar plexus block, BFI value increased by 1.79 fold at the second toe, 1.74 fold at the third toe, 1.96 fold at the fourth toe, and 1.88 fold at the little toe. For failed lumbar plexus block, no significant difference was found in BFI values at each time point after the block compared with the baseline value. BFI value of the big toe at $10 \mathrm{~min}$ after lumbar plexus block showed great power to predict block outcome with a sensitivity of $100 \%$ and a specificity of $100 \%$. The optimal cut-off value given by ROC analysis was $22.11 \mathrm{PU}$.

For successful selective FNB, ONB, and LFCNB, BFI value at each toe was not significantly increased after the block compared with the baseline value (Fig. 3).. There were no significant differences in mean arterial pressure before and after the block (Table 2).

\section{Discussion}

This study investigated the blood flow changes determined by LSCI technique after lumbar plexus block, or successful selective FNB, ONB, and LFCNB, respectively. Our results demonstrated that the BFI values were significantly increased as early as 5 min after successful lumbar plexus block, whereas no significant difference was found in BFI values after failed lumbar plexus block. BFI value of the big toe at 10 min after lumbar plexus block showed great power to predict block outcome with a sensitivity of $100 \%$ and a specificity of $100 \%$. Successful selective blockade of femoral nerve, obturator nerve, or lateral femoral cutaneous nerve did not cause BFI changes. To our knowledge, this is the first study using LSCI 
technique to identify the blood flow changes after blockade of the lumbar plexus or selective blockade of its three major branches.

Successful lumbar sympathetic block had been assessed by various objective methods. Infrared thermographic imaging was reported to be a simple and safe method for assessing successful lumbar sympathetic block. Cutaneous temperature changes at the most distal parts of the lower extremity by thermocouple probe were reported to predict the efficacy of lumbar sympathetic block in the early stage. ${ }^{78}$ However, skin temperature monitoring can be easily influenced by environmental temperature. The pulse oxymetry was introduced to measure change in pulse transit time in the lower extremity, which had been considered as an early indicator of successful lumbar sympathetic block. ${ }^{10}$ Skin conductance monitor was proved to indicate successful lumbar sympathetic block for all procedures. ${ }^{27}$ Nevertheless, the pulse oxymetry and skin conductance monitor are restricted to single-point measurement and unable to quantify overall perfusion in the involved capillary bed. Compared with these assessment methods, the superiority of $\mathrm{LSCl}$ technique lies in high temporal and spatial resolution in a wide field of view. For $\mathrm{LSCl}$ measurements, region of interests could be freely selected and the BFI values were real-time acquired. It was reported that the best place to observe peripheral vascular responsiveness was fingertip. ${ }^{22}$ As we have previously reported, the BFI values of toes were significantly increased after successful sciatic nerve block. ${ }^{20}$ Therefore, the nail bed of each toe was chosen as region of interests in this study.

In the current study, the BFI value with the highest increase was at the big toe after successful lumbar plexus block. It seems that the big toe was the ideal position to observe blood flow changes after lumbar plexus block. Accordingly, one previous study showed that the maximum change of skin temperature after successful lumbar sympathetic block was attained at the big toe when compared with the other regions in the lower extremity. ${ }^{7}$ Another study demonstrated that the greatest rise and most rapid changes in skin temperature occurred in the big toe, irrespective of the applied regional-anesthesia techniques of the lower extremity (combined femoral-nerve and sciatic-nerve block, epidural anesthesia, and spinal anesthesia). ${ }^{28}$ Areas of skin temperature increase were not totally determined by the segmental innervation area ${ }^{2228}$, which indicated that the sympathetic block territory was not corresponding to the cutaneous innervation of the blocked nerve. It could be advantageous to perform $\mathrm{LSCl}$ measurements at the big toe because this region was abundant in arteriovenous anastomoses and richly innervated by sympathetic vasoconstrictor fibers. ${ }^{29}$

Our results revealed that BFI value at each toe was not significantly increased after successful selective femoral nerve block. This observation supported the prior finding that changes of skin temperature after femoral nerve block were negligible and late. ${ }^{30}$ Sympathetic nerve fibers descend to join the lumbar plexus from the sympathetic trunk in a complex way, which has not been known in detail. ${ }^{31}$ It was reported that the femoral artery blood flow responses were not mediated by the lumbar sympathetic nerves. ${ }^{32}$ In our study, BFI value at each toe was not significantly changed after selective block of three major branches of lumbar plexus separately. This may suggest that selective block of the separate nerves at more peripheral sites have relatively little impact on sympathetic blockade. 
$\mathrm{LSCl}$ has been widely applied in clinical practice to better quantify the microcirculation perfusion in real time. ${ }^{33-36}$ Compared with the traditional assessment methods such as pinprick or cold sensory tests, LSCI measurement was objective, simple, and powerful. Traditional block assessing methods depend on patients' subjective judgement to the pinprick or cold stimuli, which is not applicable in special patient population (such as elderly patients with preoperative cognitive dysfunction, children, patients with mental problems, and patients who received peripheral nerve block after general anesthesia). LSCI technique is an alternative measurement to predict the block success of lumbar plexus with high sensitivity and specificity, but cannot indicate the success for selective FNB, ONB, and LFCNB.

Laser speckle images are generated as soon as the target is illuminated by laser source. The LSCI system takes full advantage of intuitive interface, fast speed and high accuracy of data acquisition, real-time image processing, and efficient data analysis. The system offers a friendly user interface which simplifies user operation without the need of special training. The simple structure and easy-to-understand interface for LSCI make the users achieve the desired output with minimal input. LSCI has become a valuable and powerful tool to investigate the fast change in microcirculation blood flow because of safety, wide measurement range, good reliability, high stability, and strong practicability. ${ }^{37-40}$ Patient populations, such as those with peripheral vascular disease, those receiving vasodilator drugs prior to surgery, or those with infected toenails might have altered the BFI values and thus require separate determination.

This study has several limitations. Firstly, the sample size was small. Secondly, we did not perform motor block assessment for obturator nerve. Sensory block assessment for obturator nerve was reported unreliable. ${ }^{41}$ Lastly, blood flow images were obtained at 5-min intervals post block in this study and continuous measurement would be better.

\section{Conclusions}

In conclusion, this study demonstrates that increased blood flow index measured by laser speckle contrast imaging is a reliable indicator of successful lumbar plexus block, but cannot indicate successful selective blocks of three major branches of the lumbar plexus. BFI value of the big toe at $10 \mathrm{~min}$ after lumbar plexus block could well predict block outcome with the cut-off value of $22.11 \mathrm{PU}$.

\section{Abbreviations}

ANOVA: Analysis of variance; ASA: American Society of Anesthesiologists; CCD: Charge-coupled device; FNB: Femoral nerve block; ITS: intertransverse space; LFCNB: lateral femoral cutaneous nerve block; LSCl: Laser speckle contrast imaging; ONB: obturator nerve block; PMTS: paramedian transverse scan; PU: perfusion unit; ROC: Receiver operator characteristic; ROIs: Region of interests

\section{Declarations}

\section{Ethics approval and consent to participate}


This prospective observational study was approved by Ethics Committee of Tongji Hospital (TJIRB20170401) and written informed consent was obtained from all subjects participating in the trial. The trial was registered prior to patient enrollment at clinicaltrials.gov (NCT03169517, Principal investigator: Wei Mei, Date of registration: May 25, 2017).

\section{Consent for publication}

The written informed consent was obtained from patients for publication of the individual blood flow images.

\section{Availability of data and materials}

The datasets used and analysed during the current study are available from the corresponding author on reasonable request.

\section{Competing interests}

The authors declare that they have no competing interests.

\section{Funding}

This work was supported by the grants from the National Natural Science Foundation of China (no. 81571357 and no. 31000417 to W. M.).

\section{Authors' contributions}

XW helped design the study, collect the data, analyze the results, and write the manuscript. PL helped design the study, analyze the results, and revise the manuscript. $X L$ and $L M$ helped design the study and prepare the manuscript. DW helped select the participants and collect the data. YH helped analyze the results and revise the manuscript. WM helped design the study, analyze the results, revise the manuscript, and supervise the study. All authors read and approved the final manuscript.

\section{Acknowledgements}

The authors would like to thank the nursing staff at Tongji Hospital in Wuhan, China for their assistance with the study.

\section{Author details}


${ }^{1}$ Department of Anesthesiology and Pain Medicine, Tongji Hospital, Tongji Medical College, Huazhong University of Science and Technology, Wuhan, 430030, China. ${ }^{2}$ Department of Anesthesiology, Institute of Anesthesiology and Critical Care Medicine, Union Hospital, Tongji Medical College, Huazhong University of Science and Technology, Wuhan 430022, China. ${ }^{3}$ Britton Chance Center for Biomedical Photonics, School of Engineering Sciences, Wuhan National Laboratory for Optoelectronics, Huazhong University of Science and Technology, Wuhan, China.

\section{References}

1. Touray ST, de Leeuw MA, Zuurmond WW, Perez RS. Psoas compartment block for lower extremity surgery: a meta-analysis. Br J Anaesth. 2008;101:750-60.

2. Hojer Karlsen AP, Geisler A, Petersen PL, Mathiesen O, Dahl JB. Postoperative pain treatment after total hip arthroplasty: a systematic review. Pain. 2015;156:8-30.

3. Ilfeld BM, Mariano ER, Madison SJ, Loland VJ, Sandhu NS, Suresh PJ, Bishop ML, Kim TE, Donohue MC, Kulidjian AA, Ball ST. Continuous femoral versus posterior lumbar plexus nerve blocks for analgesia after hip arthroplasty: a randomized, controlled study. Anesth Analg. 2011;113:897-903.

4. Li J, Karmakar MK, Li X, Kwok WH, Ngan Kee WD. Regional hemodynamic changes after an axillary brachial plexus block: a pulsed-wave Doppler ultrasound study. Reg Anesth Pain Med. 2012;37:1118.

5. Hingorani AP, Ascher E, Gupta P, Alam S, Marks N, Schutzer RW, Multyala M, Shiferson A, Yorkovich W, Jacob T, Salles-Cunha S. Regional anesthesia: preferred technique for venodilatation in the creation of upper extremity arteriovenous fistulae. Vascular. 2006;14:23-6.

6. Sorensen J, Bengtsson M, Malmqvist EL, Nilsson G, Sjoberg F. Laser Doppler perfusion imager (LDPI) -for the assessment of skin blood flow changes following sympathetic blocks. Acta Anaesthesiol Scand. 1996;40:1145-8.

7. Tran KM, Frank SM, Raja SN, El-Rahmany HK, Kim LJ, Vu B. Lumbar sympathetic block for sympathetically maintained pain: changes in cutaneous temperatures and pain perception. Anesth Analg. 2000;90:1396-401.

8. Park SY, Nahm FS, Kim YC, Lee SC, Sim SE, Lee SJ. The cut-off rate of skin temperature change to confirm successful lumbar sympathetic block. J Int Med Res. 2010;38:266-75.

9. Kim YC, Bahk JH, Lee SC, Lee YW. Infrared thermographic imaging in the assessment of successful block on lumbar sympathetic ganglion. Yonsei Med J. 2003;44: 119-24.

10. Joo EY, Kong YG, Lee J, Cho HS, Kim SH, Suh JH. Change in pulse transit time in the lower extremity after lumbar sympathetic ganglion block: an early indicator of successful block. J Int Med Res. 2017;45:203-10.

11. Rege A, Murari K, Li N, Thakor NV. Imaging Microvascular Flow Characteristics Using Laser Speckle Contrast Imaging. Conf Proc IEEE Eng Med Biol Soc. 2010;2010:1978-81. 
12. Miao P, Rege A, Li N, Thakor NV, Tong S. High resolution cerebral blood flow imaging by registered laser speckle contrast analysis. IEEE Trans Biomed Eng. 2010;57:1152-7.

13. Ansari MZ, Nirala AK. Monitoring capillary blood flow using laser speckle contrast analysis with spatial and temporal statistics. Optik. 2015;126:5224-9.

14. Feng N, Qiu J, Li P, Sun X, Yin C, Luo W, Chen S, Luo Q. Simultaneous automatic arteries-veins separation and cerebral blood flow imaging with single-wavelength laser speckle imaging. Opt Express. 2011;19:15777-91.

15. Ren J, Li P, Zhao H, Chen D, Zhen J, Wang Y, Wang Y, Gu Y. Assessment of tissue perfusion changes in port wine stains after vascular targeted photodynamic therapy: a short-term follow-up study. Lasers Med Sci. 2014;29:781-8.

16. Chen DF, Ren J, Wang Y, Zhao HY, Li BH, Gu Y. Relationship between the blood perfusion values determined by laser speckle imaging and laser Doppler imaging in normal skin and port wine stains. Photodiagn Photodyn. 2016;13:1-9.

17. Yang B, Yang O, Guzman J, Nguyen P, Crouzet C, Osann KE, Kelly KM, Nelson JS, Choi B. Intraoperative, real-time monitoring of blood flow dynamics associated with laser surgery of port wine stain birthmarks. Lasers Surg Med. 2015;47:469-75.

18. Ponticorvo A, Cardenas D, Dunn AK, Ts'o D, Duong TQ. Laser speckle contrast imaging of blood flow in rat retinas using an endoscope. J Biomed Opt. 2013;18:090501.

19. Okamoto $M$, Yamashita $M$, Ogata $N$. Effects of intravitreal injection of ranibizumab on choroidal structure and blood flow in eyes with diabetic macular edema. Graefes Arch Clin Exp Ophthalmol. 2018;256:885-892.

20. Wu X, Li J, Joypaul K, Bao WW, Wang D, Huang YJ, Li PC, Mei W. Blood flow index as an indicator of successful sciatic nerve block: a prospective observational study using laser speckle contrast imaging. Br J Anaesth. 2018;121:859-66.

21. Minville V, Gendre A, Hirsch J, Silva S, Bourdet B, Barbero C, Fourcade O, Samii K, Bouaziz H. The efficacy of skin temperature for block assessment after infraclavicular brachial plexus block. Anesth Analg. 2009;108:1034-6.

22. Lange KH, Jansen T, Asghar S, Kristensen PL, Skjonnemand M, Norgaard P. Skin temperature measured by infrared thermography after specific ultrasound-guided blocking of the musculocutaneous, radial, ulnar, and median nerves in the upper extremity. $\mathrm{Br} \mathrm{J}$ Anaesth. 2011;106:887-95.

23. Karmakar MK, Li JW, Kwok WH, Soh E, Hadzic A. Sonoanatomy relevant for lumbar plexus block in volunteers correlated with cross-sectional anatomic and magnetic resonance images. Reg Anesth Pain Med. 2013;38:391-7.

24. Flack S, Anderson C. Ultrasound guided lower extremity blocks. Paediatr Anaesth. 2012;22:72-80.

25. Danelli G, Ghisi D, Bellinghieri F, Borghi B, Fanelli G, Chelly JE. The nerve stimulation technique versus the loss of resistance technique for the posterior approach to lumbar plexus block: a randomized, prospective, observer-blinded, pilot study. Minerva Anestesiol. 2011;77:959-63. 
26. Dolan J, Williams A, Murney E, Smith M, Kenny GN. Ultrasound guided fascia iliaca block: a comparison with the loss of resistance technique. Reg Anesth Pain Med. 2008;33:526-31.

27. Gungor S, Rana B, Fields K, Bae JJ, Mount L, Buschiazzo V, Storm H. Changes in the Skin Conductance Monitor as an End Point for Sympathetic Nerve Blocks. Pain Medicine. 2017;18:218797.

28. Werdehausen R, Braun S, Hermanns H, Freynhagen R, Lipfert P, Stevens MF. Uniform distribution of skin-temperature increase after different regional-anesthesia techniques of the lower extremity. Reg Anesth Pain Med. 2007;32:73-8.

29. Mowafi HA. Digital skin blood flow as an indicator for intravascular injection of epinephrinecontaining simulated epidural test dose in sevoflurane-anesthetized adults. Anesth Analg. 2005;101:584-8.

30. Stevens MF, Werdehausen R, Hermanns H, Lipfert P. Skin temperature during regional anesthesia of the lower extremity. Anesth Analg. 2006;102:1247-51.

31. Feigl GC, Dreu M, Ulz H, Breschan C, Maier C, Likar R. Susceptibility of the genitofemoral and lateral femoral cutaneous nerves to complications from lumbar sympathetic blocks: is there a morphological reason? Br J Anaesth. 2014;112:1098-104.

32. Song J, Tanida M, Shibamoto T, Zhang T, Wang M, Kuda Y, Kurata Y. The Role of Lumbar Sympathetic Nerves in Regulation of Blood Flow to Skeletal Muscle during Anaphylactic Hypotension in Anesthetized Rats. PLoS One. 2016;11:e0150882.

33. Knudsen KBK, Thorup J, Strandby RB, Ambrus R, Ring LL, Ifaoui I. Laser Speckle Contrast Imaging to Evaluate Bowel Lesions in Neonates with NEC. European J Pediatr Surg Rep. 2017;5:e43-e6.

34. Ambrus R, Achiam MP, Secher NH, Svendsen MBS, Runitz K, Siemsen M, Svendsen LB. Evaluation of Gastric Microcirculation by Laser Speckle Contrast Imaging During Esophagectomy. J Am Coll Surg. 2017;225:395-402.

35. Tesselaar E, Flejmer AM, Farnebo S, Dasu A. Changes in skin microcirculation during radiation therapy for breast cancer. Acta Oncol. 2017;56:1072-80.

36. Barcelos A, Tibirica E, Lamas C. Evaluation of microvascular endothelial function and capillary density in patients with infective endocarditis using laser speckle contrast imaging and videocapillaroscopy. Microvasc Res. 2018;118:61-8.

37. Roustit M, Millet C, Blaise S, Dufournet B, Cracowski JL. Excellent reproducibility of laser speckle contrast imaging to assess skin microvascular reactivity. Microvasc Res. 2010;80:505-11.

38. Murray AK, Manning J, Moore T, Wilkinson J, Marjanovic E, Leggett S, Dinsdale G, Roberts C, Allen J, Anderson ME, Britton J, Buch M, Del Galdo F, Denton CP, Drayton T, Furlong A, Griffiths B, Hall F, Hart D, Howell K, MacDonald A, McHugh NJ, Pauling J, Shipley J, Herrick A. A Multicentre Reliability and Validity Study of Laser Speckle Contrast Imaging and Thermography in Patients with Raynaud's Phenomenon Secondary to Systemic Sclerosis. Rheumatology. 2017;56:172.

39. Mirdell R, Farnebo S, Sjoberg F, Tesselaar E. Accuracy of laser speckle contrast imaging in the assessment of pediatric scald wounds. Burns. 2018;44:90-8. 
40. Ringkamp M, Wooten M, Carson BS, Sr., Lim M, Hartke T, Guarnieri M. Laser speckle imaging to improve clinical outcomes for patients with trigeminal neuralgia undergoing radiofrequency thermocoagulation. J Neurosurg. 2016;124:422-8.

41. Bouaziz H, Vial F, Jochum D, Macalou D, Heck M, Meuret P, Braun M, Laxenaire MC. An evaluation of the cutaneous distribution after obturator nerve block. Anesth Analg. 2002;94:445-9.

\section{Tables}

Table 1 Patient characteristic data.

\begin{tabular}{lccccc}
\hline & $\begin{array}{c}\text { Successful LPB } \\
(\mathrm{n}=21)\end{array}$ & Failed LPB & Successful FNB & Successful ONB & Successful \\
& $34.0 \pm 10.7$ & $29.0 \pm 12.7$ & $42.8 \pm 20.9$ & $39.0 \pm 18.3$ & $43.2 \pm 12.5$ \\
\hline Age (years) & $16(76)$ & $2(100)$ & $5(63)$ & $2(14)$ & $7(70)$ \\
Male sex [n (\%)] & $167.2 \pm 7.5$ & $178.0 \pm 9.9$ & $168.0 \pm 3.7$ & $160.4 \pm 6.5$ & $167.1 \pm 12.5$ \\
Height (cm) & $63.0 \pm 12.7$ & $70.5 \pm 6.4$ & $62.4 \pm 9.5$ & $52.6 \pm 6.1$ & $67.3 \pm 9.2$ \\
Weight (kg) & $9(43)$ & $1(50)$ & $2(25)$ & $3(43)$ & $2(20)$ \\
ASA I [n (\%)] & $117.9 \pm 31.6$ & $111.5 \pm 16.3$ & $115.0 \pm 30.7$ & $111.1 \pm 16.0$ & $109.9 \pm 17.8$ \\
Duration of & & & & \\
surgery (min) & & & &
\end{tabular}

Data are presented as the mean \pm SD or number (proportion). LPB, lumbar plexus block; FNB, femoral nerve block; ONB, obturator nerve block; LFCNB, lateral femoral cutaneous nerve block.

Table 2 Hemodynamic data of mean arterial pressure ( $\mathrm{mmHg}$ ). 


\begin{tabular}{lccccc}
\hline $\begin{array}{l}\text { Time } \\
(\mathrm{min})\end{array}$ & $\begin{array}{c}\text { Successful LPB } \\
(\mathrm{n}=21)\end{array}$ & $\begin{array}{c}\text { Failed LPB } \\
(\mathrm{n}=2)\end{array}$ & $\begin{array}{c}\text { Successful FNB } \\
(\mathrm{n}=8)\end{array}$ & $\begin{array}{c}\text { Successful ONB } \\
(\mathrm{n}=8)\end{array}$ & $\begin{array}{c}\text { Successful LFCNB } \\
(\mathrm{n}=8)\end{array}$ \\
\hline 0 & $86.2 \pm 7.7$ & $86.5 \pm 2.1$ & $89.7 \pm 17.1$ & $87.6 \pm 13.1$ & $87.9 \pm 9.4$ \\
5 & $84.5 \pm 7.6$ & $84.0 \pm 1.4$ & $92.1 \pm 17.4$ & $88.4 \pm 16.7$ & $89.7 \pm 9.6$ \\
10 & $87.6 \pm 7.6$ & $83.5 \pm 3.5$ & $88.5 \pm 13.5$ & $88.0 \pm 14.9$ & $89.9 \pm 11.6$ \\
15 & $87.2 \pm 8.9$ & $81.5 \pm 0.7$ & $87.0 \pm 13.7$ & $89.5 \pm 18.0$ & $88.7 \pm 12.0$ \\
20 & $85.6 \pm 9.9$ & $84.5 \pm 0.7$ & $93.5 \pm 10.2$ & $89.3 \pm 13.9$ & $89.0 \pm 6.6$ \\
25 & $88.7 \pm 9.9$ & $82.0 \pm 2.8$ & $91.8 \pm 14.3$ & $90.1 \pm 15.7$ & $88.5 \pm 10.5$ \\
30 & $86.1 \pm 10.3$ & $85.0 \pm 5.7$ & $87.3 \pm 17.3$ & $87.8 \pm 16.3$ & $87.8 \pm 12.6$ \\
\hline
\end{tabular}

Data are presented as the mean \pm SD. LPB, lumbar plexus block; FNB, femoral nerve block; ONB, obturator nerve block; LFCNB, lateral femoral cutaneous nerve block.

\section{Figures}

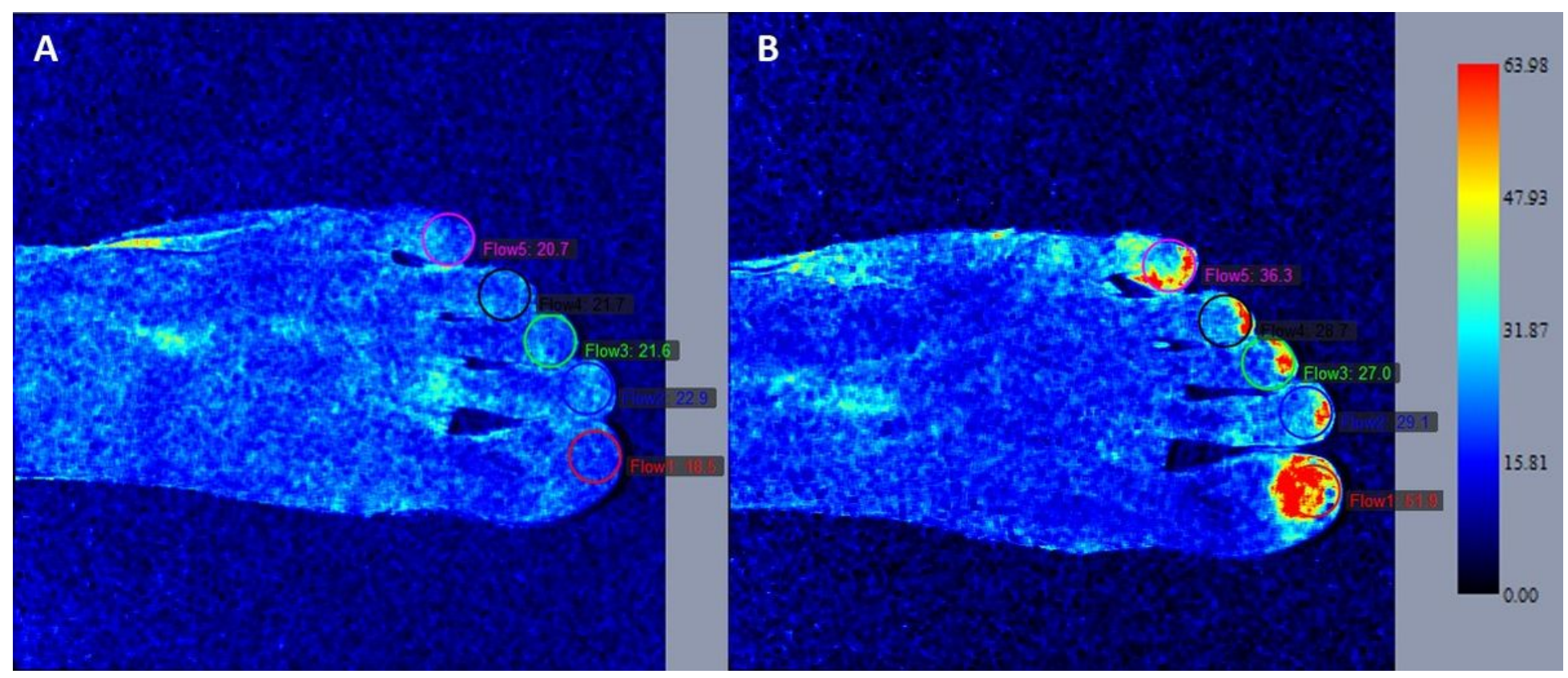

\section{Figure 1}

Blood flow images and BFI values of ROls. (A) BFI values of ROls in a 19-year-old male patient before lumbar plexus block; (B) BFI values of ROls in a 19-year-old male patient at 5 min after successful lumbar plexus block. BFI, blood flow index; ROls, region of interests. 
The great toe

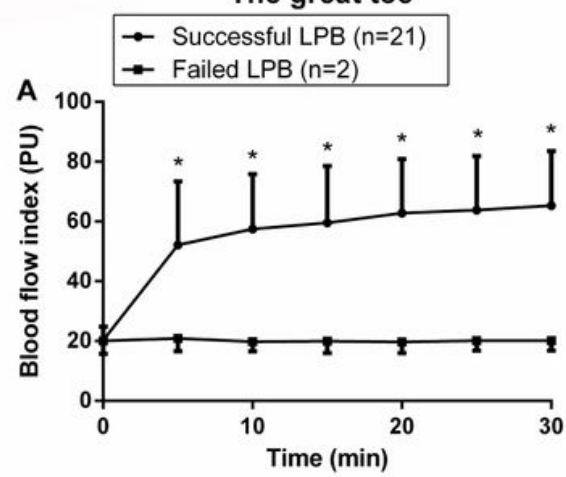

The fourth toe

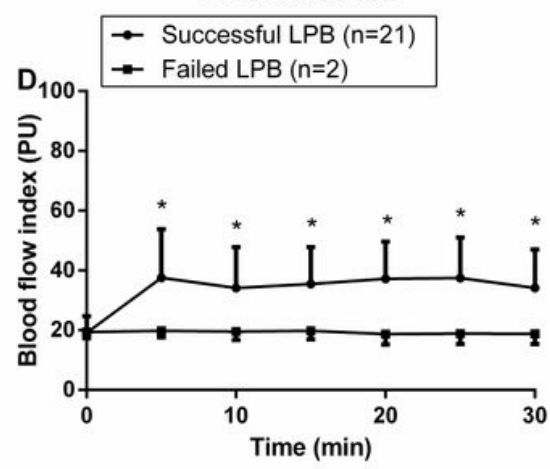

The second toe

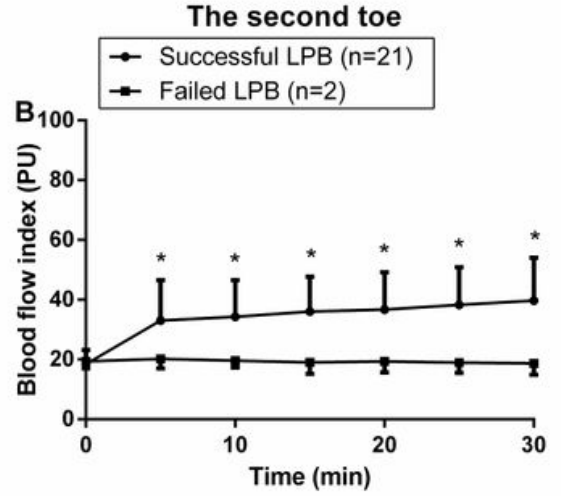

The little toe

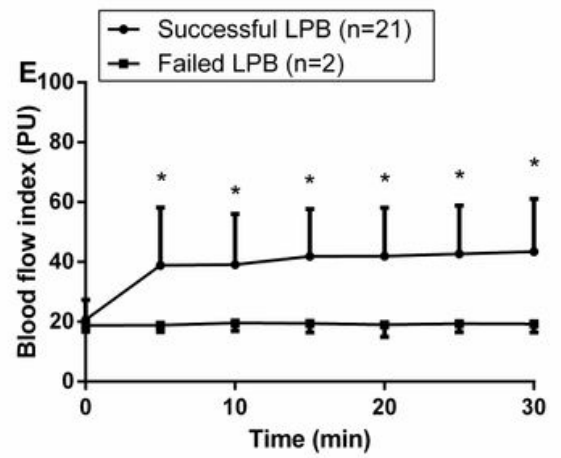

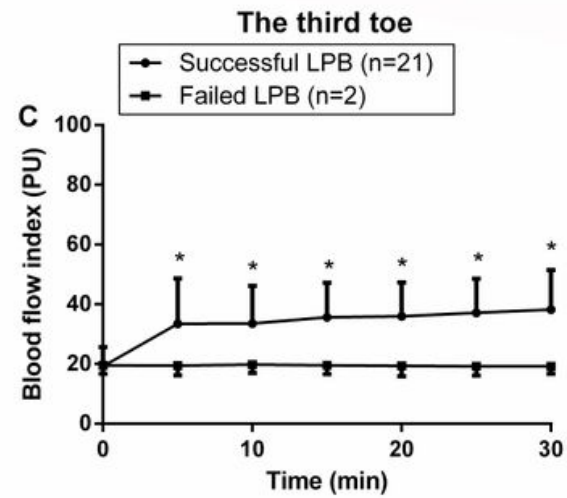

\section{Figure 2}

Blood flow index values of the toes in patients that underwent successful LPB and failed LPB. (A) BFI value of the big toe; (B) BFI value of the second toe; (C) BFI value of the third toe; (D) BFI value of the fourth toe; and $(E) B F I$ value of the fifth toe. ${ }^{\star} P \leq 0.001$ compared with the baseline value. LPB, lumbar plexus block. 
The great toe

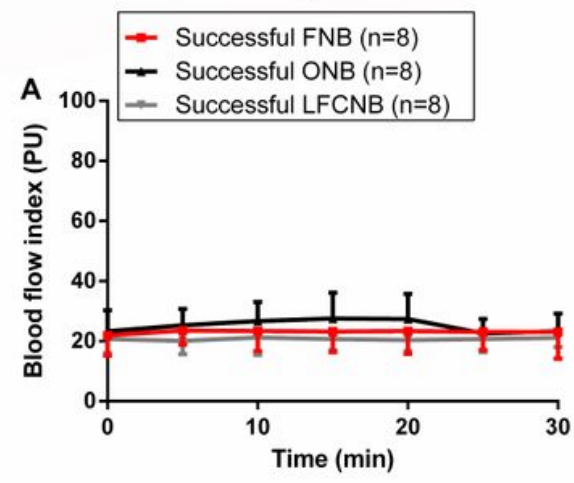

The fourth toe

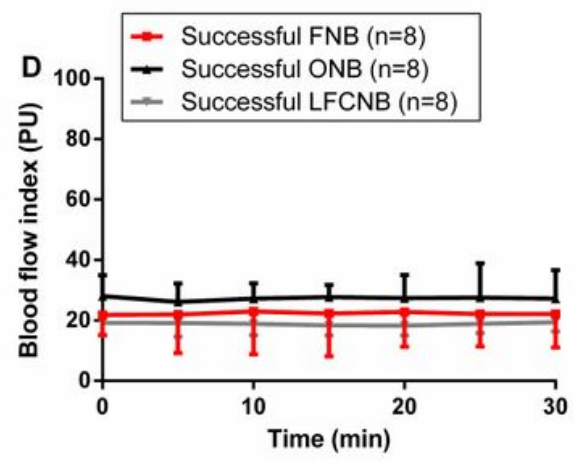

The second toe
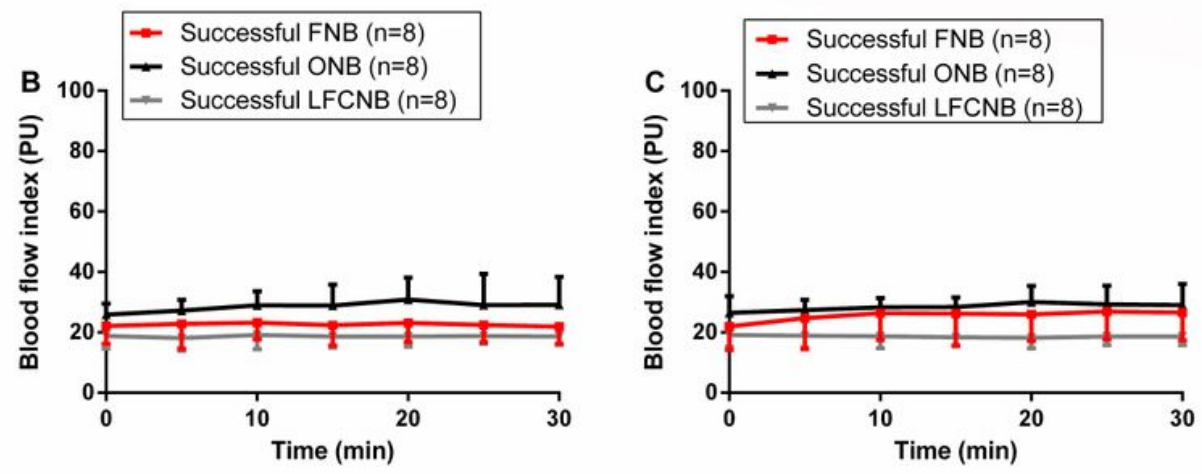

\section{Figure 3}

Blood flow index values of the toes in patients that underwent successful selective successful FNB, ONB and LFCNB. (A) BFI value of the big toe; (B) BFI value of the second toe; (C) BFI value of the third toe; (D) $B F I$ value of the fourth toe; and $(E) B F I$ value of the fifth toe. FNB, femoral nerve block; ONB, obturator nerve block; LFCNB, lateral femoral cutaneous nerve block. 